\title{
Examinee abilities and task difficulty in task-based second language performance assessment
}

\author{
John M. Norris Northern Arizona University, James D. \\ Brown, Thom D. Hudson University of Hawaii at Manoa and \\ William Bonk University of Colorado
}

\begin{abstract}
This article summarizes findings from investigations into the development and use of a prototype English language task-based performance test. Data included performances by 90 examinees on 13 complex and skills-integrativetasks, a priori estimations of examinee proficiency differences, a priori estimations of task difficulty based on cognitive processing demands, performance ratings according to task-specific as well as holistic scales and criteria, and examinee self-ratings. Findings indicated that the task-based test could inform intended inferences about examinees' abilities to accomplish specific tasks as well as inferences about examinees' likely abilities with a domain of tasks. Although a relationship between task difficulty estimates and examinee performances was observed, these estimates were not found to provide a trustworthy basis for inferring examinees ${ }^{+}$likely abilities with other tasks. These findings, as well as study limitations, are further discussed in light of the intended uses for performance assessment within language education, and recommendations are made for needed research into the interaction between task features, cognitive processing and language performance.
\end{abstract}

\section{Introduction}

Long and Norris (2000: 60) distinguish task-based from other forms of language performance assessment as follows:

[T]ask-based language assessment takes the task itself as the fundamental unit of analysis motivating item selection, test instrument construction, and the rating of task performance. Task-based assessment does not simply utilize the real-world task as a means for eliciting particular components of the language system, which are then measured or evaluated; instead, the construct of interest is performance of the task itself.

For some time, interest has been expressed in using such a task-based assessment approach to address certain inferential purposes found in second/foreign language education contexts (Long, 1985; Brindley, 1994). For example, applications of task-based assessment have been proposed in conjunction with academic/specific purposes (Robinson and Ross, 1996), content-based (Byrnes, 1998), survival-skills (Clark and Grognet, 1985), adult immigrant (Norton, 2000) and task-based

Address for correspondence: John M. Norris, 633 N. Pine Cliff, Flagstaff, AZ 86001, USA: email: norrisjm@earthlink.net

Language Testing 200219 (4) 395-418 


\section{Examinee abilities and task difficulty}

(Long and Crookes, 1993) language instruction, where teachers and others often want to know whether examinees can accomplish particular target tasks or task types with the language knowledge and abilities they have been learning.

Despite such interest on the part of second language (L2) educators, language testers have argued (e.g., Shohamy, 1995; McNamara, 1996; Skehan, 1998) that explicitly task-based (or task-centred) inferences probably should not serve as the focus of language performance assessment because, among other shortcomings, a task-based approach:

- does not provide any basis for making interpretations beyond the particular task/test context;

- cannot hope to simulate all of the factors that define actual language use situations; and

- may elicit performances that depend on abilities or knowledge unrelated to language per se.

Likewise, concerns have been voiced in the educational measurement literature regarding the potentially detrimental effects on learning of relying solely on task-centred performance assessment (e.g., Linn and Burton, 1994; Messick, 1994; Popham, 1997). However, for a variety of purposes in a number of education contexts, and especially for classroominternal use by teachers, it has also been argued that complex, integrative, open-ended, task-specific tests are necessary for meeting actual inferential demands (e.g., relevant interpretations about what learners know and can do) and for achieving the intended consequences of assessment (e.g., fostering students' abilities to do things with the knowledge they have acquired, beyond the simple display of that knowledge on tests) (see Wiggins, 1993; Delandshere and Petrosky, 1998; Khattri et al., 1998; Eisner, 1999; Haertel, 1999). In language education as well, there persists an apparent need for task-based assessment that can inform warranted inferences about learners. Language teachers, subject matter teachers, language learners, potential employers and others frequently want to know whether or not, or the degree to which, a leamer can utilize language in order to accomplish specific target communication tasks, ranging from the survival-related to the job-specific or academic. At the same time, available language tests may provide poor surrogates for the kinds of assessment procedures and evaluative criteria that are relevant for judging the extent to which an L2 user can actually accomplish a given task (Jacoby and McNamara, 1999; Douglas, 2000; Douglas and Myers, 2000; Norris, 2001).

In response to these perceived inferential needs, and with an eye towards addressing some of the problems above, we have engaged in a research project that focuses on the development of prototype taskbased performance tests (which we call the ALP) for use by teachers 
in language classrooms and programs. Our primary goal in the project was to model and research, under the practical testing conditions associated with university-level second and foreign language education in the USA, the development and implementation of assessment instruments and procedures which enable:

1) elicitation of examinee performances on complex, skills-integrative and goal-oriented tasks that require language use for their accomplishment;

2) evaluation of performances on individual tasks according to specific criteria for task accomplishment (as defined by knowledgeable informants, not test-developers) and evaluation of performances across test tasks according to criteria for holistic examinee abilities; and

3) interpretation of examinees' abilities with respect to particular tasks as well as a domain of tasks.

Investigations associated with points (1) and (2) are reported elsewhere (Norris et al., 1998; Norris, 2001; Brown et al., in press). In this article, we address aspects of our research related to point (3), focusing on findings for the following questions:

- To what extent are interpretations about examinees' abilities with individual tasks and with a domain of tasks warranted?

- To what extent is task 'difficulty', as estimated by several cognitive processing factors ostensibly engaged by test tasks, systematically associated with task performance?

\section{Methodology}

In conjunction with our prototype test development project, and in order to begin to address the preceding research questions, performance data were collected from examinees with widely ranging language proficiencies as they attempted to accomplish 13 complex and skills-integrative tasks using English. Performances were evaluated both by raters and by the examinees themselves using several rating scales. Outcomes were then compared among groups of examinees and categories of assessment tasks, in order to evaluate the validity of interpretations about examinees' abilities and task difficulty. Due to space constraints, only minimal descriptions of the participants, tasks, rating scales and assessment procedures are provided. Details are available in Brown et al. (in press).

\section{Participants}

Ninety volunteer examinees (compensated for participation) were recruited in two college settings, 60 at the University of Hawaii (UH) 


\section{Examinee abilities and task difficulty}

and 30 at the Kanda University of International Studies (KUIS) in Japan. At UH, English language users were drawn from four different groups:

- intermediate proficiency learners studying in a universitypreparatory English language program (HELP);

- university-matriculated international undergraduate and graduate students fulfilling English language requirements (ELI);

- advanced L2 users of English studying or working at the university (ESL); and

- first language users of English studying or working at the university (NS).

At KUIS, volunteers were recruited from similar groups of language users (students beginning their university English language studies were assigned to the HELP group, students at advanced levels of university English language training were assigned to the ELI group, etc.). Thus, all 90 volunteers were categorized a priori according to four English language levels. We posited that each of these levels would represent ability differences in accomplishing the range of tasks targeted by the ALP.

Participants from each ability level were assigned in approximately equal proportions to complete one of three ALP forms (J, P, Q). The 30 participants at KUIS completed Form J, and the 60 participants at UH completed either Form P or Form Q (described below). Table I shows participation at each ability level on each form. Unequal representation across the four levels reflects the pattern of participants responding to the call for volunteers and efforts to maintain roughly equivalent distributions for each of the three test forms.

\section{Test tasks, forms, and administration procedures}

Test items originated within a large pool of complex, skills-integrative performance tasks that require English language use in order to accomplish finite communication goals, such as might be found within

Table 1 Distribution of participants on each ALP form by a priori ability level

\begin{tabular}{lcccc}
\hline Ability level & Form $J(n)$ & Form P $(n)$ & Form Q $(n)$ & Total $(N)$ \\
\hline HELP & 7 & 6 & 4 & 17 \\
ELI & 15 & 15 & 18 & 48 \\
ESL & 5 & 6 & 5 & 16 \\
NS & 3 & 3 & 3 & 9 \\
Total $(N)$ & 30 & 30 & 30 & 90 \\
\hline
\end{tabular}


an adult ESL curriculum (see Norris $e t$ al., 1998). These research tasks were developed as prototypical examples for task-based performance assessment, with the principal inferential purpose of informing interpretations about an individual examinee's ability to use language in order to accomplish a specific task under target language use circumstances. In order for interpretations of this sort to be warranted, tasks were designed to simulate target language use situations (as were performance rating criteria, see below). Thirteen such tasks were operationalized in the current research project. While these prototypical tasks reflected generic content and thematic areas (such as 'health' or 'at the restaurant'), it should be clear that such broad descriptive labels were not intended to define a performance or ability domain to which individual task performances would extrapolate (see Norris et al., 1998). Inferences according to this kind of content-based extrapolation (e.g., from an observed performance on a 'health' task to likely performance on another 'health' task) were not investigated in the current project.

In addition to task-specific inferences, we also sought to better understand the relationship between task performance outcomes and certain cognitive processing factors that might be associated with the target tasks. That is, we were interested in the extent to which a minimal set of cognitive factors, ostensibly engaged to varying degrees and in varying combinations by tasks within our test item pool, might serve as a basis for making predictions about an examinee's ability to accomplish other tasks on the test and a range of tasks related to those on the test. In light of this secondary intended inferential purpose, test tasks were further developed and sampled into test forms according to several additional parameters.

Drawing on work by Skehan $(1996 ; 1998)$ among others, a basic set of three cognitive factors (briefly: complexity of the language code to be processed, complexity of the cognitive operations involved and processing demands associated with the required communication activities) had been identified as a possible framework for categorizing tasks within our original item pool according to so-called task 'difficulty' (see Norris et al., 1998). The purpose for this framework was to establish one means for comparing tasks with each other, based on the extent to which they engaged these particular types of cognitive factors during performance. The basic assumption was that tasks engaging such factors to a greater extent would be more 'difficult' for a particular population of examinees than would other tasks; by extension, examinees who were able to accomplish higher difficulty tasks (those engaging the cognitive factors to a greater extent) would also likely be able to accomplish lower difficulty tasks (those engaging the cognitive factors to a lesser extent).

In order to initiate investigations into the use of this additional 
inferential framework, the 13 ALP tasks were developed such that each cognitive factor would be represented in a robust manner, thereby ensuring that if different combinations of the posited factors did indeed translate into performance differences among examinees, such differences would be detectable in performance outcomes. Table 2 shows that, for each of the three cognitive factors there are either two minuses or two pluses associated with each task, indicating that each of two task features (shown in the second row) for any one factor were hypothesized to be either absent or sufficiently engaged within a task to play a substantial cognitive role in performance (the identification of cognitive factors and task features is discussed in Norris et al., 1998; Brown et al., in press). Combinations of cognitive factors (i.e., the a priori 'difficulty' estimates according to this framework) associated with the 13 tasks selected for the ALP resulted in two tasks which engaged all factors (Type 6 tasks), five tasks which engaged two of the three factors (Type 4 tasks) and six tasks which engaged one of the three factors (Type 2 tasks). Table 2 shows that all possible combinations of the three cognitive factors were represented by the collection of 13 tasks.

In order to maintain consistency with assessment contexts in which tests such as the ALP might be used, it was decided that test administration time would total no more than two hours, approximately the amount of time provided for end of semester final exams in foreign language courses at UH. Based on typical performance times that had been identified during pilot testing, the 13 ALP tasks were sampled into three test forms consisting of seven items each, as shown in Table 3. Identical combinations of the posited cognitive factors were represented by the seven tasks on each of the three forms, and tasks with input that was primarily aural or primarily visual were counterbalanced equivalently

Table 2 Matrix of cognitive processing factors posited fot prototype ALP tasks

\begin{tabular}{|c|c|c|c|c|c|c|c|c|}
\hline \multicolumn{2}{|c|}{ Task } & \multirow{2}{*}{$\begin{array}{l}\text { Type: } \\
\text { Difficulty } \\
\text { a priori }\end{array}$} & \multicolumn{2}{|c|}{$\begin{array}{l}\text { Code } \\
\text { complexity }\end{array}$} & \multicolumn{2}{|c|}{$\begin{array}{l}\text { Cognitive } \\
\text { complexity }\end{array}$} & \multicolumn{2}{|c|}{$\begin{array}{l}\text { Communicative } \\
\text { demand }\end{array}$} \\
\hline Aural & Visual & & Range & $\begin{array}{l}\text { Input/ } \\
\text { output } \\
\text { sources }\end{array}$ & $\begin{array}{l}\text { Input/ } \\
\text { output } \\
\text { organized }\end{array}$ & $\begin{array}{l}\text { Input/ } \\
\text { output } \\
\text { available }\end{array}$ & Mode & $\begin{array}{l}\text { Response } \\
\text { level }\end{array}$ \\
\hline A9 & F9 & 6 & + & + & + & + & + & + \\
\hline E21 & $\mathrm{F} 7$ & 4 & + & + & + & + & - & - \\
\hline $\mathrm{B} 20$ & $\mathrm{~B} 20$ & 4 & + & + & - & - & + & + \\
\hline E20 & E22 & 4 & - & - & + & + & + & + \\
\hline A20 & $\mathrm{C} 14$ & 2 & + & + & - & - & - & - \\
\hline F5 & A18 & 2 & - & - & + & + & - & - \\
\hline C15 & A21 & 2 & - & - & - & - & + & + \\
\hline
\end{tabular}


across the three forms. Item B20 served as an anchor task, appearing on all three test forms, while six of the 13 tasks appeared on two of the forms, and the remaining six tasks appeared on a single form.

Each of the 90 examinees was individually administered one of the three ALP forms, with each session lasting between 60 and 110 minutes, depending on how quickly an examinee completed the seven test tasks. In order to ensure comparable task performance conditions for all examinees, as well as for the two testing locations in Hawaii and Japan, detailed test instructions and administration procedures had been developed, pilot-tested and revised (see Brown et al., in press). Test administrators at each site followed these procedures for the presentation and clarification of test and task instructions, the provision of task realia and response formats at appropriate points, and the collection of video and audio recordings of performances as well as written performance products.

\section{Performance rating scales and procedures}

Examinee performances were evaluated both by examinees themselves, directly following test administration, and subsequently by three raters using two unique rating scales. For self-rating, a simple instrument had been developed, asking three questions about each of the seven tasks examinees had just completed:

- To what extent they were familiar with the task.

- How well they thought they had performed the task; and

- How easy/difficult they found the task.

A graphic element, which examinees had seen in the instructions for each task, was used to remind examinees of their performances, and responses were indicated on a three-point Likert scale.

Performances by each of the 90 examinees were also rated by three

Table 3 Task sampling for ALP Forms J, P and Q

\begin{tabular}{|c|c|c|c|c|c|c|c|c|}
\hline \multicolumn{3}{|c|}{ Form J } & \multicolumn{3}{|c|}{ Form $\mathrm{P}$} & \multicolumn{3}{|c|}{ Form $Q$} \\
\hline Task & Type & L2 input & Task & Type & L2 input & Task & Type & L2 input \\
\hline$E 20$ & 4 & aural & E22 & 4 & visual & $\mathrm{E} 20$ & 4 & aural \\
\hline A21 & 2 & visual & $\mathrm{C} 15$ & 2 & aural & A21 & 2 & visual \\
\hline B20 & 4 & mixed & B20 & 4 & mixed & B20 & 4 & mixed \\
\hline F5 & 2 & aural & A18 & 2 & visual & F5 & 2 & aural \\
\hline $\mathrm{Fg}$ & 6 & visual & $\mathrm{A} 9$ & 6 & aural & F9 & 6 & visual \\
\hline F7 & 4 & visual & F7 & 4 & visual & $\mathrm{E} 21$ & 4 & aural \\
\hline A20 & 2 & aural & A20 & 2 & aural & $\mathrm{C} 14$ & 2 & visual \\
\hline
\end{tabular}


university-level ESL teachers hired for the purpose. Prior to rating, the teachers had engaged in minimal training, which consisted of acquainting themselves with the 13 tasks and the rating rubrics. However, extensive practice rating and rater norming was not undertaken; training was minimized in order to simulate constraints that are often associated with the implementation of performance assessment in language programs.

Raters judged examinee performances (using recordings and collected task products as the performance data) according to two sets of rating scales. Following the principal inferential purpose for the ALP, the first scale had been developed to assess the extent to which an individual examinee could accomplish a given target task. Specific performance rating criteria for each task had been identified and developed by a team of informants (not the test-developers) knowledgeable about both the target tasks and the population of interest in the current study (for details, see Norris, 2001). These task-dependent rating criteria, and the resulting rating rubrics, thus varied among the 13 ALP tasks, depending on the particular elements associated with success on each task. For example, for one task, raters judged examinees' success in ordering a pizza with correct ingredients, size, etc., while for another, raters focused on an examinee's success in locating information within a library catalogue. Specific descriptors were developed for three levels of performance on each task (an inadequate performance, an able/ successful performance and an adept performance), and these three levels were converted into five-point scales, with intermediate levels included between each descriptor to allow for the rating of borderline performances.

In addition to these inferences about examinees' accomplishment of particular tasks, we also wanted to investigate the potential use of the ALP test for informing inferences about examinees' abilities underlying the performance of a range of target tasks; such inferences would be used to make predictions from full-test performance to likely abilities with a domain of related tasks. Accordingly, a second rating approach had been developed as a means for holistically evaluating examinee ability, based on performance on the entire ALP test. Three scales were employed, one each representing the three cognitive factors that were ostensibly engaged in varying degrees and combinations across the range of ALP tasks. Holistic performance evaluations according to these taskindependent scales were assigned by raters after they had inspected an examinee's entire test performance and had completed assigning ratings according to the task-dependent rating scales. Raters utilized five-point Likert scales (again ranging from inadequate to adept) to indicate their impressions of an examinee's overall performance abilities with respect to each of the three cognitive factors. 
To summarize, the following data formed the evidentiary basis for investigations in the current study:

1) a priori estimations of likely task accomplishment abilities for 90 examinees, based on four English language proficiency levels;

2) a priori estimations of task 'difficulty' for each of 13 ALP tasks, based on combinations of three cognitive processing factors;

3) examinee self-ratings of task familiarity, performance and ease/difficulty for each ALP task;

4) rater evaluations of examinee success on each ALP task, based on task-dependent rating scales;

5) rater evaluations of holistic examinee ability for three cognitive factors, based on task-independent rating scales.

\section{Summary and discussion of findings}

Several analytic techniques were employed in order to investigate relationships among the data sources listed above. Readers are directed to Brown et al. (in press) for the results of all primary analyses, including: descriptive, multi-faceted Rasch model, correlational, multivariate and scalar analyses. We present here only a summary of the associated findings in the form of selected secondary analyses, in order to examine the extent to which initial evidence supports the inferences investigated in the current project.

A preliminary concern for basing any inferences on the outcomes of the ALP test was the extent to which rating procedures would lead to consistent evaluations of examinee performances, especially given the fact that raters received very little training in the use of the scales and rubrics. As reported in detail in Brown et al. (in press), for each of the two rating approaches employed, relatively high reliability estimates were observed for the three raters, ranging from $r_{x x^{\prime}}=0.88$ to $r_{x x^{\prime}}=0.98$ for the 13 task-dependent scales and from $r_{x x^{\prime}}=0.91$ to $r_{x x^{\prime}}=0.94$ for three task-independent scales. In addition, low standard error values accompanied by good model fit statistics from multifaceted Rasch model analyses (see Table 4), provided support for the use of examinee ratings (averaged across the three raters) to inform subsequent inferences. Although a higher separation value for the task-independent ratings did suggest that raters were utilizing slightly different scale points in judging holistic examinee abilities (see measure statistics in Table 4), this small difference could be easily corrected in light of the otherwise consistent rating patterns. 


\section{Examinee abilities and task difficulty}

\section{Task-specific performance abilities}

The principal purpose for the prototype ALP assessment was to model a framework for making interpretations about an examinee's abilities to accomplish particular L2 tasks (i.e., one intended use for the test was to inform test-users about the extent to which an examinee could employ language in order to accomplish a given task under nontest circumstances). Thus, assessment tasks were operationalized with substantial fidelity to target language use situations, such that taskspecific language performances could be elicited and evaluated according to the particular criteria associated with the accomplishment of such tasks under nontest circumstances. Task-dependent rating scales provided a systematic means for applying such criteria.

Several sources of evidence gathered in the current project provided indications about the extent to which interpretations specific to examinee performances on particular tasks may be warranted. First, we observed that minimally trained raters were able to consistently apply the 13 unique task-dependent rating scales in identifying those performances which fulfilled the specific criteria associated with task accomplishment. Table 5 shows high simple agreement ratios between each pair of raters on each ALP task and overall for this dichotomous decision (i.e., whether the task was accomplished or not). Some variability in agreement levels indicates that raters were more consistent in applying accomplishment criteria for some of the tasks, and the sources for this variability should be addressed in future investigations. However, that three minimally trained raters were generally in close agreement in evaluating the accomplishment of 13 different tasks according to 13 distinct sets of criteria provides initial support for the use of ALP outcomes to inform the fundamental interpretation about whether or not an examinee is able to accomplish a particular task.

Table 4 Rasch model estimates of rater performance on two rating scales

\begin{tabular}{lcccc}
\hline Rater & Measure & S.E. & Infit Zstd & Outfit Zstd \\
\hline Task-dependent & & & & \\
1 & -0.01 & 0.04 & 0 & 0 \\
2 & 0.01 & 0.04 & 1 & 0 \\
3 & -0.00 & 0.04 & 1 & 0 \\
Task-independent & & & & \\
1 & -0.23 & 0.11 & -1 & -1 \\
2 & -1.28 & 0.12 & 0 & 0 \\
3 & -1.05 & 0.11 & 1 & 1 \\
\hline
\end{tabular}


Next, we compared average task-dependent performance ratings for examinees grouped at each of the four a priori English language ability levels. We had hypothesized that examinees would be differentially successful in accomplishing the collection of 13 ALP tasks in the following pattern: NS > ESL > ELI > HELP. In addition, we had posited that patterns of task accomplishment would prove systematic, with some of the tasks only being accomplished by the NS group, other tasks being accomplished only by the NS and the ESL groups, etc. If these independent predictions of differential ability levels matched the actual task accomplishment patterns identified by raters using the task-dependent criteria, then this would provide concurrent validational support for the use of such scales and criteria to inform interpretations about individual examinee performances.

In order to examine the relationship between predicted ability levels and task-dependent performance ratings, implicational scalar analyses were conducted following Guttman (1950). Table 6 summarizes the scalar analysis comparing average task-dependent ratings for each of the ability groups on each of the 13 ALP tasks. A performance rating of ' 3 ' on the task-dependent scales had been set as the criterion level for task accomplishment (i.e., a rating of ' 3 ' indicated that a performance met the minimal criteria identified by informants for a given task to be considered accomplished). Table 6 shows not only that each ability level group on average accomplished increasing numbers of tasks in the order predicted, but also that patterns of success were clearly implicational. Following Guttman's (1950) and Dunn-Rankin's (1983) parameters for interpreting a scale to be implicational (i.e., a minimum coefficient of reproducibility of 0.90 and a

Table 5 Simple agreement on task accomplishment decisions

\begin{tabular}{lllll}
\hline Task & $N$ (ratings) & Faters 1 and 2 & Raters 2 and 3 & Raters 1 and 3 \\
\hline E20 & 60 & 0.92 & 0.92 & 0.85 \\
A21 & 60 & 0.80 & 0.80 & 0.82 \\
B20 & 90 & 0.92 & 0.83 & 0.89 \\
F5 & 60 & 0.88 & 0.93 & 0.95 \\
F9 & 60 & 0.95 & 0.92 & 0.95 \\
E21 & 30 & 0.90 & 0.93 & 0.93 \\
C14 & 30 & 0.93 & 0.93 & 1.00 \\
E22 & 30 & 0.97 & 0.97 & 0.93 \\
C15 & 30 & 0.93 & 0.90 & 0.97 \\
A18 & 30 & 0.93 & 0.87 & 0.93 \\
A9 & 30 & 0.80 & 0.80 & 0.73 \\
F7 & 60 & 0.85 & 0.87 & 0.92 \\
A20 & 60 & 0.93 & 0.95 & 0.92 \\
All tasks & 630 & 0.90 & 0.89 & 0.91 \\
\hline
\end{tabular}


minimum coefficient of scalability of 0.60 ), scalar statistics underscore the obvious implicational pattern in the data $(\mathrm{CR}=0.92, \mathrm{CS}=$ 0.67 ), with only a few exceptions ( 2 of 13 tasks for 2 of 4 ability groups reflected unpredicted average scoring patterns). This convergence of two independent data sources (i.e., ability groupings and task performances) to form the predicted implicational pattern further supports the use of ALP outcomes to inform task-specific interpretations about individual examinees.

Finally, we investigated the performance patterns associated with individuals' task-dependent ratings on each of the three ALP forms independently. We had posited that, if the 13 task-dependent scales and criteria were functioning as intended (i.e., as accurate indications of an examinee's ability to accomplish a given task), then accomplishment decisions for individual performances on each of the seven test items would result in implicational scales for each ALP form. Were implicational scales not found in the performance data, then this would leave open the possibility that the task-dependent scales and criteria were not accurate indications of actual examinee abilities. Table 7 indicates that, using a best-fit approach to scaling, each of the three ALP forms showed scalar patterns at the task accomplishment criterion rating level of ' 3 ', with both Forms $J$ and Q surpassing implicational scale parameters, and Form P showing

Table 6 Average group success on 13 ALP tasks, based on a priori English language ability levels

\begin{tabular}{lccll}
\hline Task & NS & ESL & ELI & HELP \\
\hline E20 & 1 & 1 & 1 & 1 \\
A20 & 1 & 1 & 1 & 1 \\
C15 & 1 & 1 & 1 & 1 \\
A21 & 1 & 1 & 1 & 0 \\
B20 & 1 & 1 & 1 & 0 \\
A18 & 1 & 0 & 1 & 0 \\
E21 & 1 & 1 & 0 & 0 \\
E22 & 1 & 1 & 0 & 0 \\
F5 & 1 & 1 & 0 & 0 \\
C14 & 0 & 1 & 0 & 0 \\
F7 & 1 & 0 & 0 & 0 \\
A9 & 1 & 0 & 0 & 0 \\
F9 & 1 & 0 & 0 & 0 \\
Total $\{13)$ & 12 & 9 & 6 & 3 \\
\hline
\end{tabular}

Note: A ' 1 ' for a given task indicates that examinees at the corresponding English ability level were assigned on average a rating of 3 or greater according to the task-dependent rating criteria for that task. $A$ ' $O$ ' indicates that examinees were assigned on average a rating of less than 3 . 
only a slightly lower than expected CR value (although only 0.03 points below the scalar minimum of 0.90 ).

Initial evidence gathered in the current project seems to support the use of the ALP tasks and task-dependent rating scales for informing interpretations about an individual examinee's abilities to accomplish particular tasks under target language use circumstances. Naturally, in operational assessment contexts, especially where important decisions are tied to such inferences, validation of such uses for this kind of task-based assessment would necessitate several further lines of inquiry, including minimally the observation of examinees actually engaging in such target tasks outside of the testing context. In addition, the relationship between task accomplishment criteria identified by expert informants and those criteria that examinees set for themselves in performing and completing a task would be beneficially investigated (perhaps via further use of examinee introspection). In the current project, we found a highly variable relationship, task by task, between examinees' perceptions of how well they performed a given task and raters' judgements of their performances on that task (Pearson $r$ ranging from 0.27 to 0.76 across the 13 ALP tasks). Thus, although raters were consistently able to make judgements about task accomplishment using criteria that varied from task to task, and these judgements were obviously supported by a priori predictions of examinee ability differences, it remains unclear to what extent an examinee's own understanding of the expectations and difficulties associated with a task may play a role in task performance and accomplishment.

\section{Holistic performance abilities}

In addition to interpretations about an examinee's abilities with individual tasks, a secondary inferential purpose for the ALP test was to inform interpretations about an examinee's holistic abilities with respect to a domain of tasks. Thus, although the various ALP items and the rating criteria associated with their accomplishment might be used to effectively estimate examinees' task-specific abilities, we were unsure to what extent patterns of ability would emerge from

Table 7 Implicational scale statistics for three ALP forms

\begin{tabular}{llll}
\hline Scale statistics & Form J & Form P & Form Q \\
\hline CR & 0.96 & 0.87 & 0.91 \\
CS & 0.82 & 0.63 & 0.69 \\
\hline
\end{tabular}

Note: Statistics show the scalar properties across individual performances on each form, using a rating of ' 3 ' as the criterion for analysis. 
performances on the entire set of test tasks. Given evidence for systematicity in overall performances, we had posited that full-test outcomes might be used for estimating an examinee's likely abilities with a domain of related tasks from which test items had been sampled. Several sources of performance data provided evidence regarding the extent to which the task-based assessment approach modelled in the current project may inform such interpretations.

Task-independent rating scales provided one tool for estimating an examinee's holistic abilities. Raters utilized the three scales (code command, cognitive operations, communicative adaptation) to evaluate an examinee's overall abilities (i.e., based on full-test performances) in dealing with the three types of cognitive processing factors engaged across the range of seven tasks appearing on each ALP form. We had posited that raters would consistently differentiate among examinees according to these cognitive factors, and that the resulting holistic ratings would be systematically related to a priori English language ability levels. As detailed in Brown et al. (in press), it was unclear based on data from the current project to what extent raters were able to distinguish among the individual cognitive factors themselves in evaluating examinees' abilities (and this should serve as a focus of future research). However, multi-faceted Rasch model analyses of outcomes from the three task-independent ratings per examinee revealed a high person separation index (4.98 logits, corresponding to a reliability of 0.96 ) for the examinees facet, as well as a wide range of examinee measure values (from -6.14 to 8.40 logits) and low standard errors, suggesting that combined outcomes of the task-independent ratings could consistently identify holistic performance differences among the 90 examinees.

In order to investigate whether such estimates of holistic performance might inform warranted interpretations about examinees' actual abilities in dealing with the cognitive demands of tasks like those found on the ALP test, we compared average task-independent ability estimates among the four a priori examinee ability levels. Once again, we had posited that these four level groups would exhibit differential holistic levels of ability in dealing with the cognitive demands of the ALP tasks. Furthermore, we assumed that these differences would be evident in task-independent ratings based on all three ALP forms, even though examinees within a single ability level group completed different subsets of test items on the different forms. Figure 1 shows that the average task-independent ability estimates for each of the four examinee ability levels differed widely and consistently in the order predicted; note that none of the $95 \%$ confidence intervals surrounding the mean estimates overlaps with any of the others.

Additionally, if holistic ratings according to the task-independent 


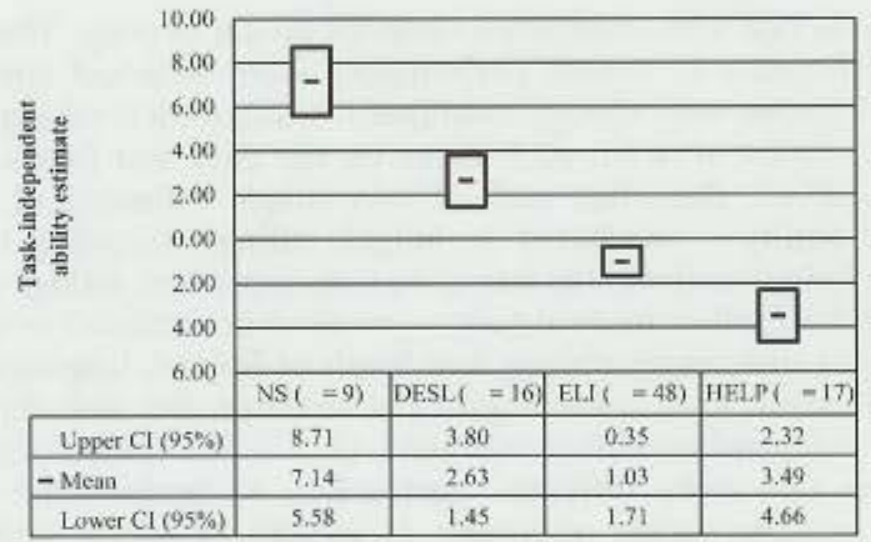

Figure 1 Average Rasch model examinee ability measures for task-independent ratings

scales were to inform interpretations about an examinee's likely abilities to accomplish a range of target tasks, then such ratings would obviously need to be demonstrably related to judgements of examinees' performances on particular target tasks. In order to investigate this relationship, we first calculated a further set of overall ability estimates based on combined performance ratings for each examinee according to the task-dependent scales. Multi-faceted Rasch model analyses showed for these scales as well that examinees spanned a wide range of ability levels according to full-test performance patterns, and that ability differences could be reliably estimated (person separation index $=3.70$, corresponding to a reliability of 0.93 ). Figure 2 shows that, as was the case for holistic ability estimates according to the task-independent scales, average task-dependent values differed widely and consistently among the four $a$ priori examinee ability level

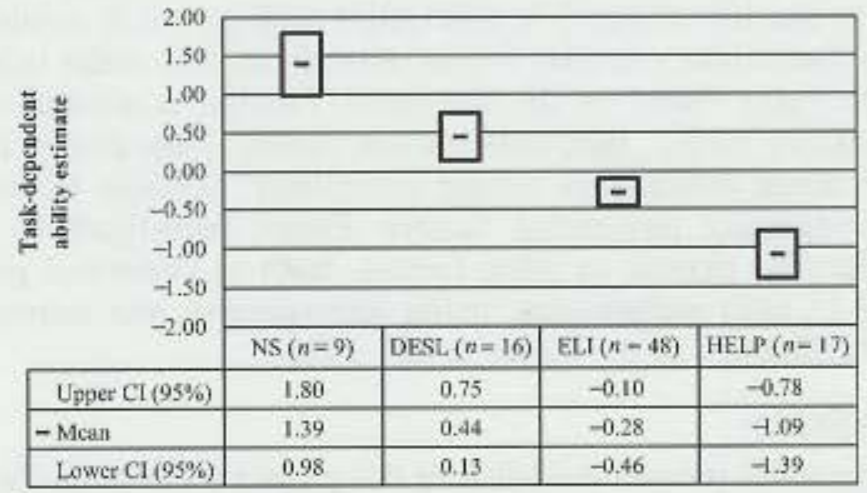

Figure 2 Average Rasch model examinee ability measures for task-independent ratings 
groups; note that $95 \%$ confidence intervals do not overlap. These consistent differences in overall performance were observed among the four level groups even though examinees within each level completed differing subsets of seven ALP items on the three test forms.

We observed, then, that each of two unique estimates of overall examinee ability - one based on holistic ratings according to three cognitive factors and another based on task-dependent ratings specific to criteria for each of thirteen tasks - resulted in predicted systematic performance differences among four levels of English language users. A high Pearson correlation coefficient between the task-dependent and task-independent ability estimates $(r=0.92)$ further suggested that these two very different approaches to performance rating resulted in very similar estimates of overall examinee ability. In addition, examinees' average self-ratings of their own performances showed a moderately strong relationship with the task-independent holistic ability estimates $(r=0.77)$.

These findings provide initial concurrent and criterion-related validational support for the use of holistic ability estimates (based on the task-independent rating scales) to inform interpretations about differences in examinees' likely abilities to accomplish tasks from the same domain as those found on the ALP test. However, once again, in operational assessment contexts, the use of such holistic ability estimates for making generalizations to an individual's likely abilities within a domain of related tasks would necessitate further validation inquiry. Specifically, a predictive mechanism relating holistic performance estimates to particular tasks or task types in the domain would first be required, representative test tasks would need to be systematically sampled from the domain according to this mechanism and subsequent predictions would then need to be verified on the basis of further performance data on a second set of related tasks from within the domain. Such domain-referenced sampling and inferencing was not investigated in this initial project, but it would prove crucial in operational contexts where tests are used to make inferences beyond the tasks found on the test itself. Finally, it remains unclear to what extent raters' task-independent ability judgements may be related to actual differences among examinees' abilities to deal with the three cognitive processing factors. Future investigations should address the role played by such factors, both in examinee performances and in rater judgements, using introspective and retrospective research techniques (e.g., Royer et al., 1993).

\section{Task difficulty}

A final inferential process modelled in this project focused on the notion of 'task difficulty'. As detailed in Norris et al. (1998), we were interested 
Table 8 Performance 'difficulty' estimates for 13 tasks

\begin{tabular}{lccc}
\hline Task & $\begin{array}{c}\text { A priori cognitive } \\
\text { task type }\end{array}$ & $\begin{array}{c}\text { Task-dependent } \\
\text { Rasch item measure }\end{array}$ & $\begin{array}{c}\text { Task-dependent } \\
\text { mean rating }\end{array}$ \\
\hline E20 & 4 & -1.56 & 3.91 \\
A20 & 2 & -1.07 & 3.77 \\
C15 & 2 & -0.85 & 3.51 \\
A21 & 2 & -0.54 & 3.22 \\
B20 & 4 & -0.11 & 3.01 \\
A18 & 2 & 0.01 & 3.01 \\
F5 & 2 & 0.12 & 2.56 \\
E22 & 4 & 0.18 & 2.64 \\
F7 & 4 & 0.30 & 2.22 \\
E21 & 4 & 0.51 & 2.71 \\
A9 & 6 & 0.51 & 2.56 \\
C14 & 2 & 1.08 & 1.99 \\
F9 & 6 & 1.42 & 1.55 \\
\hline
\end{tabular}

in exploring whether combinations of several cognitive factors (code complexity, cognitive complexity and communicative demand) triggered by certain task features might be systematically associated with differing degrees of success in task performance. 'Difficulty' was therefore operationalized in the current project as a set of predictions regarding the likely interaction between task features and examinee abilities, resulting in the following three categories of tasks:

- Type 2: any one of the three cognitive factors engaged;

- Type 4: any two of the three factors engaged; and

- Type 6: all three of the factors engaged).

Thus, if examinees who accomplished more 'difficult' tasks (i.e., those engaging more factors) were consistently found to perform less 'difficult' tasks at equivalent or higher levels of success, then performances on a representative sample of such tasks might be used to inform generalizations about an examinee's abilities within a domain of tasks which engaged cognitive factors in similar ways.

In order to enable direct comparisons among the 13 tasks and the three task types, an examinee- and test-form-free estimate of the performance patterns associated with each task was provided by Rasch model item measures based on the task-dependent ratings (which revealed a high reliability of 0.99 in distinguishing among the 13 tasks). Table 8 shows the item measure for each task (in logits), as well as the task type designation and the average raw-score task-dependent performance rating. While overall patterns suggest that tasks engaging more cognitive factors resulted in generally lower rated levels of performance, this was not a 


\section{Examinee abilities and task difficulty}

categorical distinction. Several tasks differed widely from expected performance levels: Task E20 (a Type 4 task) showed the lowest item measure value (i.e., the highest rated levels of performance), while task C14 (a Type 2 task) showed the second highest item measure value (i.e., the second lowest rated levels of performance). Several other tasks also showed item measure values that differed from the predicted order of difficulties. Given these observations, it could not be concluded in this study that the different combinations of cognitive factors ostensibly engaged by ALP tasks were systematically associated with degrees of performance success on particular tasks (as estimated according to the task-dependent rating scales).

Examinees' perceptions of the ALP tasks (in the form of selfratings) offered useful points of comparison for deliberations about the relationship between task 'difficulty' and performance success. Item measure estimates for the 13 ALP tasks showed only a moderate relationship with average examinee self-ratings of the ease/difficulty with which the tasks were performed (Pearson $r=-0.60$ ). However, examinees' average self-ratings of their familiarity with the 13 tasks showed a much stronger relationship with the Rasch model item measure estimates $(r=-0.87)$. Furthermore, for the two tasks that resulted in the most unpredictable levels of performance success, E20 and C14, self-ratings suggest that examinees' levels of familiarity may have played an important role in their ability to perform the two tasks, with E20 receiving the second highest familiarity rating and $\mathrm{C} 14$ receiving the second lowest familiarity rating.

Further analyses were undertaken in order to investigate whether full-test performance patterns might generally be associated with the three cognitive task types. Both average Rasch model item measure

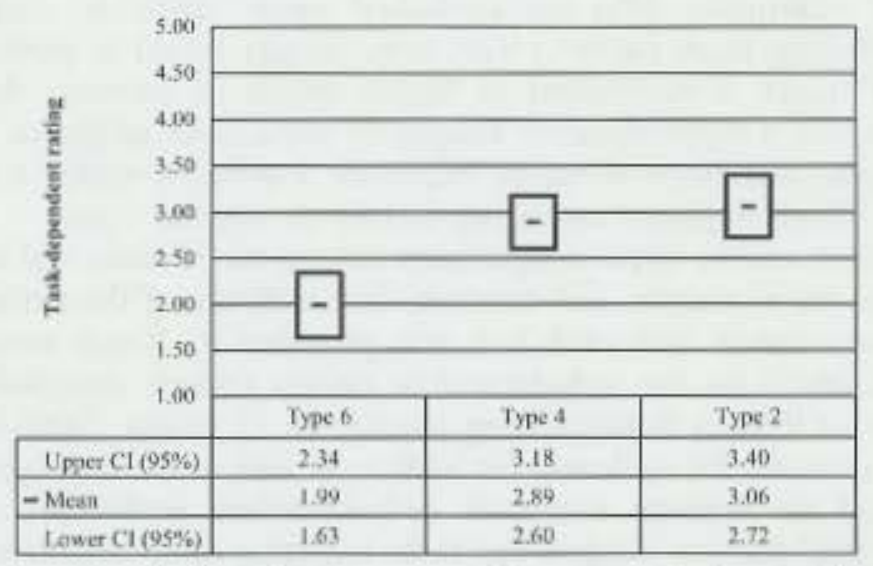

Figure 3 Average task-dependent raw score performance rating by cognitive task type 
estimates and average raw-score task-dependent ratings (adjusted for nonequivalent examinee sampling; see Brown et al, in press) revealed the following pattern of performance success: Type $2>$ Type $4>$ Type 6 . However, Figure 3 shows that while Type 6 tasks showed obvious substantial raw score performance rating differences from the other two types, Type 4 tasks differed only slightly on average from Type 2 tasks (with largely overlapping $95 \%$ confidence intervals).

We also investigated the extent to which average performance ratings for each of the four English ability levels might be systematically associated with differing performances on the three cognitive task types. Table 9 shows that average raw-score performance ratings did not consistently reflect the pattern predicted among the task types within each of the four ability levels (i.e., Type 6 showing the lowest ratings and Type 2 showing the highest). While Type 6 tasks did turn out to have the lowest average performance ratings for all four groups, Type 2 tasks resulted in lower average performance ratings than did Type 4 tasks for both the NS and ESL groups. The predicted order in performance patterns for the three task types was only observed within the two lowest ability groups.

Nevertheless, it is of interest that, when a criterion score of ' 3 ' was set as the minimum level for task accomplishment (as directed by expert informants), a clear relationship was found in the order predicted by both task type and examinee ability level. That is, based on average scores, only the NS group succeeded on all three task types; the ESL group succeeded on Type 4 and Type 2 tasks; the ELI group succeeded only on Type 2 tasks; and the HELP group did not succeed on any of the task types. Of course, such average performance ratings should not be interpreted as categorical differences among the ability groups in terms of individual examinees' levels of task accomplishment. Table 10 shows the proportions of examinees receiving an average rating of ' 3 ' or better on each of the three task types for each of the four ability groups. Once again, overall patterns of accomplishment did not necessarily indicate the

Table 9 Average success for ability level groups by cognitive task types (mean raw score performance rating for each examinee group on each task type in parentheses)

\begin{tabular}{|c|c|c|c|c|c|c|}
\hline Task type & $\begin{array}{l}\text { NS } \\
(n=9)\end{array}$ & $\begin{array}{l}\text { ESL } \\
(n=16)\end{array}$ & $\begin{array}{l}\text { ELI } \\
(n=48)\end{array}$ & $\begin{array}{l}\text { HELP } \\
(n=17)\end{array}$ & $\begin{array}{l}\text { Task-dependent } \\
\text { mean rating }\end{array}$ & $\begin{array}{l}\text { Rasch model } \\
\text { item measure }\end{array}$ \\
\hline Type 2 & $1(4.01)$ & $1(3.58)$ & $1(3.01)$ & $0(2.20)$ & 3.06 & -0.21 \\
\hline Type 4 & $1(4.83)$ & $1(3.73)$ & $0(2.62)$ & $0(1.84)$ & 2.89 & -0.13 \\
\hline Type 6 & $1(3.86)$ & $0(2.17)$ & $0(1.76)$ & $0(1.47)$ & 1.99 & 0.97 \\
\hline
\end{tabular}

Note: $\mathrm{A}^{\prime} 1$ ' or ' 0 ' score was assigned to each examinee group on each task type using minimum rating of 3 as a criterion for success. 


\section{Examinee abilities and task difficulty}

same degrees of success for all examinees within a given level. Patterns also showed unpredictable differences in proportions of examinees accomplishing each of the three task types within the lowest and highest predicted ability groups.

In summary, initial evidence from this study did not support the use of the cognitive processing factors - as operationalized in our original task difficulty framework - for the estimation of eventual performance difficulty differences among test tasks. While there was some indication that average performance levels associated with the three cognitive task types differed in predicted ways, these differences did not extend to individual tasks. What is more, evidence suggests that examinees may have been responding to tasks in idiosyncratic ways, in particular as a result of their familiarity with both task content and task procedures. As such, the use of this framework for the purposes of generalizing from examinee performances on ALP test tasks to a domain of related tasks may not be warranted. Of course, the current study did not seek to determine empirically the extent to which the hypothesized cognitive factors played a role in examinee performances on the small set of test tasks as operationalized on the ALP. As such, it remains unclear to what extent the tasks actually:

- engaged the cognitive processing factors in the predicted combinations; and

- elicited particular factors to the degrees predicted.

What is clear, however, is that the task-dependent criteria identified by expert informants and used to evaluate specific task performances had little to do with the cognitive factors ostensibly involved in performing the tasks. That any relationship was observed between these disparate variables suggests to us that future research that explores more directly the role played by cognitive processing within examinee performances may prove worthwhile (for a related discussion, see Norris and Ortega, in press). Such future research would also profit from investigation of:

- a larger set of tasks related by posited underlying processing factors;

Table 10 Proportion of examinees succeeding on three cognitive task types

\begin{tabular}{lllll}
\hline Task type & NS $(n=9)$ & ESL $(n=16)$ & ELI $(n=48)$ & HELP $(n=17)$ \\
\hline Type 2 & 0.89 & 0.69 & 0.50 & 0.13 \\
Type 4 & 1.00 & 0.63 & 0.48 & 0.06 \\
Type 6 & 0.78 & 0.25 & 0.10 & 0.12 \\
\hline
\end{tabular}


- the manipulation of individual tasks by adding or subtracting features associated with processing difficulty (see Robinson, 2001); and

- the role that may be played in performances by various types of cultural, linguistic and task procedural knowledge.

\section{Conclusions}

While limitations in research methods (for details, see Brown et al., in press) prevent definitive conclusions, we hope that the findings reported here provide some basis and impetus for future investigations into the development, use and validation of task-based tests. We found that careful simulations of target L2 communication tasks could effectively elicit a wide range of examinee performances, and that minimally trained raters could consistently utilize two very different rating scales and sets of criteria for judging these performances. Average performance patterns based on both rating scales also clearly reflected hypothesized differences among four a priori ability levels of examinees. This evidence provides initial support for the use of the prototype ALP test and task-dependent ratings for informing interpretations about an examinee's ability to accomplish specific target tasks, as well as the use of task-independent ratings for distinguishing among examinees according to their general abilities in performing the range of test tasks.

We did not find that the cognitive processing factors as operationalized in our original task difficulty framework proved particularly useful for estimating actual performance difficulty differences among test tasks. As such, initial evidence does not support the use of this framework as an inferential basis for generalizing from examinees' performances on specific tasks to likely abilities with related tasks. Nevertheless, the observation of some degree of relationship between the cognitive factors and task-dependent performance evaluations (i.e., those based on 'real-world' criteria identified by external informants) suggests a fruitful area for research on language performance assessment. One way to begin to address the problems of performance assessment noted at the beginning of this article is to look much more carefully into the actual relationship between task features and the behaviours they elicit, to investigate the role played by cognitive variables in determining such behaviours, and to relate these findings to inferences that need to be made on the basis of language performance assessment (for discussion, see Nichols and Sugrue, 1999; Norris and Ortega, in press). Indeed, we would argue that a central focus for research on all types of language performance assessment - including assessment which informs the constructcentred as well as the task-centred ends of the inferential continuum 


\section{Examinee abilities and task difficulty}

- should be to better understand how tasks are actually accomplished (in both cognitive and 'real-world' terms) and what makes a given task more or less 'difficult' for different examinees. Without a better understanding of performance along these lines, the validity of inferences about language learners' abilities (of whatever sort) will remain in question (Mislevy et al., 1998).

In closing, we would like to reiterate that the task-based assessment approach modelled in this project was intended for use by practitioners within language classrooms and programs, where inferences need to be made about examinees' abilities with respect to particular target tasks or task types. We hope it is clear that we are not suggesting that this approach should supplant numerous other approaches to test development and use that have arisen in response to a variety of inferential demands in L2 education. On the contrary, and as we have stated elsewhere (Brown and Hudson, 1998; Norris, 2000), we think that those test instruments and procedures should be employed which best match actual assessment purposes (including, even, 'alternative' purposes, an issue much belaboured at a recent AAAL colloquium). In all likelihood, virtually all language education contexts will require the use of a variety of assessment alternatives in order to meet the actual inferential demands (and intended consequences) that exist, including task-centred as well as construct-centred tests, discretepoint items as well as extended performance tasks and very specific as well as very global scoring procedures (see, e.g., the integrative assessment model in Khattri et al., 1998: 33). As such, rather than ignoring or dismissing the variety of actual purposes for assessment, language testers should respond by dealing with the admittedly thorny issues of test development, use and validation in order to better inform those inferences that need to be made.

\section{Acknowledgements}

This is a revised version of a paper presented at the 22nd Language Testing Research Colloquium, Vancouver, in the colloquium 'Putting tasks to the test'. We thank Cathie Elder for organizing the colloquium and inviting our participation. This research was funded in part by a Language Resource Centers Program grant from the US Department of Education.

\section{References}

Brindley, G. 1994: Task-centred assessment in language learning: the promise and the challenge. In Bird, N., Falvey, P., Tsui, A., Allison, D. and $\mathrm{McNeill,} \mathrm{A.,} \mathrm{editors,} \mathrm{Language} \mathrm{and} \mathrm{learning:} \mathrm{papers} \mathrm{presented} \mathrm{at} \mathrm{the}$ 
Annual International Language in Education Conference (Hong Kong, 1993). Hong Kong: Hong Kong Education Department, 73-94.

Brown, J.D. and Hudson, T.D. 1998: The alternatives in language assessment: advantages and disadvantages. TESOL Quarterly 30, 653-75.

Brown, J.D., Hudson, T.D., Norris, J.M. and Bonk, W. in press: An investigation of second language task-based performance assessments. Honolulu: University of Hawaii Press.

Byrnes, H. 1998: Constructing curricula in collegiate foreign language departments. In Byrnes, $\mathrm{H}$, editor, Learning foreign and second languages. New York: The Modern Language Association of America, 262-96.

Clark, J.L.D. and Grognet, A.G. 1985: Development and validation of a performance-based test of ESL 'survival skills'. In Hauptman, P., LeBlanc, R. and Wesche, M., editors, Second language performance testing. Ottawa: University of Ottawa Press, 89-110.

Delandshere, G. and Petrosky, A. 1998: Assessment of complex performances: limitations of key measurement assumptions. Educational Researcher 27, 14-24.

Douglas, D. 2000: LSP assessment criteria: where do they come from? Paper presented at the Second Asian Language Assessment Research Forum, January $11-12,2000$.

Douglas, D. and Myers, R. 2000: Assessing the communication skills of veterinary students: whose criteria? In Kunnan, A.J., editor, Faimess and validation in language assessment: selected papers from the 19th Language Testing Research Colloquium, Orlando, Florida. New York: Cambridge University Press, 60-81.

Dunn-Rankin, P. 1983: Scaling methods. Hillsdale, NJ: Lawrence Erlbaum.

Eisner, E.W. 1999: The uses and limits of performance assessment. Phi Delta Kappan 80, 658-60.

Guttman, L. 1950: The basis of scalogram analysis. In Stouffer, S.A., editor, Measurement and prediction. Princeton, NJ: Princeton University.

Haertel, E.H. 1999: Performance assessment and education reform. Phi Delta Kappan 80, 662-66.

Jacoby, S. and McNamara, T. 1999: Locating competence. English for Specific Purposes 18, 213-41.

Khattri, N., Reeve, A. and Kane, M. 1998: Principles and practices of performance assessment. Mahwah, NJ: Lawrence Erlbaum.

Linn, R.L. and Burton, E. 1994: Performance-based assessment: implications of task specificity. Educational Measurement: Issues and Practice $13,5-15$.

Long, M.H. 1985: A role for instruction in second language acquisition: task-based training. In Hyltenstam, K. and Pienemann, M., editors, Modelling and assessing second language acquisition. Clevedon, Avon: Multilingual Matters, 77-100.

Long, M. and Crookes, G. 1993: Units of analysis in syllabus design. In Crookes, G., and Gass, S., editors, Tasks in a pedagogical context: integrating theory and practice. Clevedon: Multilingual Matters, 9-54.

Long, M.H. and Norris, J.M. 2000: Task-based language teaching and 


\section{Examinee abilities and task difficulty}

assessment. In Byram, M., editor, Encyclopedia of language teaching. London: Routledge, 597-603.

MeNamara, T. 1996: Measuring second language performance. New York: Longman.

Messick, S. 1994: The interplay of evidence and consequences in the validation of performance assessments. Educational Researcher 23, 13-23.

Mislevy, R.J., Steinberg, L.S., Breyer, R.J., Almond, R.G. and Johnson, L. 1998: A cognitive task analysis, with implications for designing a simulation-based performance assessment. Los Angeles, CA: University of California, National Center for Research on Evaluation, Standards, and Student Testing (CRESST).

Nichols, P. and Sugrue, B. 1999: The lack of fidelity between cognitively complex constructs and conventional test development practice. Educational Measurement: Issues and Practice 18, 18-29.

Norris, J.M. 2000: Purposeful language assessment. English Teaching Forum 38, 18-23.

2001: Identifying rating criteria for task-based EAP assessment. In Hudson, T.D. and Brown, J.D., editors, A focus on language test development: expanding the language proficiency construct across a variety of tests. Honolulu: University of Hawaii Press, 163-204.

Norris, J.M., Brown, J.D., Hudson, T.D. and Yoshioka, J. 1998: Designing second language performance assessments. Honolulu: University of Hawaii Press.

Norris, J.M. and Ortega, L. (in press). Defining and measuring L2 acquisition. In Doughty, C. and Long, M.H., editors, Handbook of second language acquisition, London: Blackwell.

Norton, B. 2000: Writing assessment: language, meaning, and marking memoranda. In Kunnan, A.J., editor, Faimess and validation in language assessment: selected papers from the 19th Language Testing Research Colloquium, Orlando, Florida. New York: Cambridge University Press, 20-29.

Popham, W. 1997: What's wrong - and what's right - with rubrics. Educational Leadership, October, 72-75.

Robinson, P. 2001: Task complexity, task difficulty, and task production: exploring interactions in a componential framework. Applied Linguistics $22,27-57$.

Robinson, P. and Ross, S. 1996: The development of task-based testing in English for academic purposes contexts. Applied Linguistics 17, 455-76.

Royer, J.M., Cisero, C.A. and Carlo, M.S. 1993: Techniques and procedures for assessing cognitive skills. Review of Educational Research $63,201-43$.

Shohamy, E. 1995: Performance assessment in language testing. Annual Review of Applied Linguistics 15, 188-211.

Skehan, P. 1996: A framework for the implementation of task-based instruction. Applied Linguistics 17, 38-62.

— 1998: A cognitive approach to language learning. Oxford: Oxford University Press.

Wiggins, G. 1993: Assessing student performance: exploring the purpose and limits of testing. San Francisco, CA: Jossey-Bass. 